\title{
De comment est (re)fait l'espace-entre : A Cidade dos Objectos [La Ville des Objets] (Augusto Alves da Silva)
}

\author{
Helena Pires*
}

\begin{abstract}
Résumé
L'exercice sémiotique que nous proposons dans ce contexte repose sur une conception ontologique et épistémologique de l'espace qui, dans une certaine mesure, se rapproche de la notion d'espace parcouru de Michel de Certeau. L'auteur s'intéresse aux «énonciations pédestres », aux pratiques de l'espace qui sont étrangères à une grammaire universalisante. Cette dimension de l'espace étant celle que nous chercherons à aborder, l'objectif de cet essai est de centrer notre attention sur les objets qui s'interposent sur les trajets sémiotiques en énonciation. Nous considérons que ces mêmes trajets sont composés de réseaux de relations hétérogènes, incorporés par des acteurs humains et non humains. Les contributions de Latour, de Donna Haraway et de Deleuze et de Guattari, entre autres, sont, elles, considérées fondamentales pour une élucidation réflexive sur la nature de ces relations. L'espace s'ouvrant, dès lors, à la possibilité de la coexistence, nous chercherons à relater la coexistence matérielle des différents corps, des différents acteurs, des manières particulières comme les objets transbordent de sens inusités et se transforment en éléments créatifs qui produisent indéfiniment l'espace, en même temps qu'ils sont produits par lui et partagent sa nature ineffable.
\end{abstract}

mots-clés : sémiotique sociale ; sémiotique de l'espace ; objets ; performativité

Sémiotique sociale de l'espace

«The study of space as a semiotic phenomenon suggests that the meaning of unspace, as
a sign, is generally understood in relation to other concerns. In everyday life space is
rarely considered for its independent qualities, but is more generally taken as a
category of conceptions that act as background to other objects and relationships»
(Gaines, 2006: 173).

Dans le cadre de l'analyse sémiotique, l'espace est communément compris, en répliquant les mots en épigraphe, comme « une catégorie de conceptions dont la fonction est de servir de fond à d'autres objets et relations ». Ce qui voudrait dire, d'après Gaines, et en convoquant le pragmatisme de Peirce, que sa dimension de priméité est niée à l'espace : ce qui est comme il est indépendamment de toute autre chose $e^{1}$. Renvoyé au second plan, l'espace est assumé, tout simplement, sans explication sur son propre rôle dans la construction du sens. Il est fréquent de nous retrouver confrontés à une compréhension de l'espace qui le confond simplement avec la zone entre les objets. Une définition selon laquelle l'espace est traduit par l'« ensemble des relations qui unissent les individus dans leur environnement » (Paul-Levy et Segaud, 1983) se superpose au contraire à une telle conception statique

\footnotetext{
*Université du Minho, CECS (Centre d'Études de Communication et Société) hpires@ics.uminho.pt

${ }^{1}$ Rappelons que selon la théorie du signe de Charles Peirce, avec la priméité, s'articulent également la secondéité (ce qui est comme il est relatif à quelque chose) et la tiercéité (ce qui est comme il comme un médiateur entre deux éléments)
} 
et limitée de l'espace. L'espace est ainsi, dès le début, un espace habité, un espace d'action et de négociation sur les manières possibles de passer, d'orienter, de limiter et, en dernière analyse, de construire la connaissance.

C'est dans l'ouverture à ces agencements et à tant d'autres que nous proposons ici un bref essai sémiotique de l'espace. L'espace qu'il sera donc intéressant de convoquer pour objet de réflexion et d'analyse est déjà et intrinsèquement un espace performatif, un espace de redéfinition permanente de proximités et de grandes distances, de différences, un espace déterminant du sens et qui, comme tel, en tant que manière de faire le sens survenir, s'affirme dans le contrepoint d'un espace supposé où le sens survient. Avant toute chose, ce bref essai souhaite rendre compte d'une notion d'espace qui refuse l'assomption d'un sujet passible de se constituer comme centre édifiant de la représentation, selon les termes d'un point de vue unique et unificateur qui organise l'univers (re)connu. Nous suggérons ainsi un déplacement de l'espace abstrait et géométrique vers l'espace vécu et qui, dans cette mesure, constitue des relations qui sont fondées à l'intérieur de l'espace.

$\mathrm{Au}$ lieu d'une sémiotique de l'espace qui présuppose la perspective d'un sujet délimité de l'extérieur, nous opterons pour une sémiotique sociale de l'espace, ce qui signifie nécessairement une sémiotique matérielle, c'est-à-dire, des aménagements que les différents acteurs, humains et non humains, favorisent lors de la construction de trajectoires et de (ré)aménagements des relations. Nous prendrons en particulier les objets comme des extensions de l'humain, à la manière d'Edward Hall (1966) entre autres, investis d'une performativité qui s'entrelace avec l'espace et dans l'espace.

\section{Espace performatif}

L'étude d'une vision sémiotique déterminée de l'univers est favorisée par la représentation en tant que mode d'accès au phénomène des relations comprises. La conscience de l'expérience, dans le temps et dans l'espace, est stimulée dans le cadre de la représentation par la possibilité d'y transformer les aspects les plus triviaux de l'espace quotidien en éléments d'une certaine signifiance. D'autre part, l'espace de représentation se constituant comme une instance d'édification de la connaissance, ce qui revient à dire d'une certaine vision du monde, il sera important de s'interroger sur les mécanismes de reproduction culturelle, mais également sur le jeu de tension sociale auquel l'espace s'ouvre d'une manière particulière. Il ne s'agit pas ici de démêler, à la manière chomskyenne, des compétences spécifiques, des formes d'orientation programmatique qui cherchent à ancrer tant l'espace de la représentation que l'espace représentationnel (niveaux syntagmatique et paradigmatique de la signification).

En s'inspirant de la proposition d'Ian Roderick (2001), cet essai souhaite, avant tout, accentuer les limites qui sont redéfinies dans la mobilité et l'immuabilité inhérentes à la performativité sémiotique, comprise comme constituant génératif de la production et de la contre-production de la 
connaissance. D'après l'auteur, il s'impose de distinguer la notion de performativité de celle avancée, notamment par Chomsky, attendu que pour ce dernier, la performance, définie comme la mise à jour ou l'usage du langage dans des situations concrètes, n'est reconnue que comme fondée sur une compétence légitimatrice que cette même performance présuppose également. Au contraire, ce sont précisément les erreurs de compétence générative programmatique, les pratiques apparemment peu importantes, les gestes qui suggèrent d'exercer les limites du représentationnel, les variations imprévisibles que nous chercherons à relever. Plus que de dévoiler la manière dont la trajectoire privilégiée se construit dans l'espace, il s'agira par conséquent de révéler la façon dont un espace déterminé agence, d'une manière particulière, l'impossibilité d'une trajectoire absolument dicible.

\section{Performativité des objets et espace-entre, chez Augusto Alves da Silva}

«... l'espace est la possibilité de la coexistence... »(Kant)

Avec Augusto Alves da Silva, photographe dont le travail a récemment (octobre 2009-février 2010) été exposé à Serralves sous la forme d'une synthèse rétrospective (Ensaio sobre o Optimismo [Essai sur l'optimisme]), la «construction de situations-type $»^{2}$ reconnaissables par le spectateur, c'est-à-dire la production d'images qui convoquent et provoquent certains stéréotypes, de l'art et de la vie, se présente comme un recours créatif surprenant. Attirant dès le début l'attention sur l'apparente neutralité qu'il accentue de manière illusoire, en renforçant dans un premier plan de lecture l'évident et le lieu commun, son travail finit par gagner une force persuasive particulière, en invitant le spectateur à se livrer à une espèce de jeu des différences. L'implication avec l'image est stimulée par la recherche des « détails qui démentent et contredisent les associations les plus pressées » ${ }^{3}$.

$\mathrm{La}$ «condition insoumise» de l'artiste le mène notamment à prendre pour objet central du regard les espaces vides et inutiles, de même que les objets et les situations insignifiantes qui composent les paysages (in)visibles de la vie quotidienne. A Cidade dos Objectos [La Ville des Objets] (1991) constitue une série particulièrement révélatrice de l'importance de penser l'espace, et dans ce cas précis, l'espace urbain, compris dans sa nature matérielle, en tant que phénomène qui se (re)fait à chaque pas, dans l'expérience toujours innovatrice du regard : «Un lampadaire, une affiche publicitaire, une cabine téléphonique, une clôture de chantiers, un panneau de la circulation, un banc de jardin apparaissent avec des paysages urbains, des quartiers sociaux, des monuments, des bâtiments abandonnés, en tant que puissants éléments de signification $»^{4}$.

À partir de l'analyse desdites images photographiques, il est important de s'interroger : De quelle manière l'espace perçu s'ouvre-t-il (ou se ferme-t-il) à la liberté de mouvement ? Comment le

\footnotetext{
${ }^{2}$ Voir João Fernandes, "Contra o documento: a fotografia e o vídeo de Augusto Alves da Silva", in Augusto Alves da Silva, Ensaio sobre o Optimismo (Catalogue de l'Exposition à Serralves), 2009.

${ }^{3}$ Idem.

${ }^{4}$ Idem, p. 13.
} 
jeu de modulations qui produit un espace vide déterminé se produit-il, une fois simultanément dédoublé dans sa valeur de séparation («terre de personne») et de convivialité (« espace commun et neutre de la rencontre »)? (Simmel). Comment l'(in)différenciation sémiotique que les différents acteurs favorisent entre eux a-t-elle lieu? De quelle manière les objets entament-ils un certain type de connexion alors qu'ils sont envisagés comme des éléments non humains avec lesquels sont établis des associations rénovées ? De quelle manière la recombinaison d'éléments hétérogènes est-elle suggérée ? De quel type de réassociations ou regroupements les images rendent-elles compte ? Par le biais de quel mouvement en particulier ? Quelles nouvelles associations sont-elles favorisées ? Quelles nouvelles formes de cohabitation?

Pour chercher à répondre à ces questions et à d'autres, ce à quoi le parcours de l'exercice proposé mènera, nous nous appuierons sur l'acception ample du social, proposée par Latour (ANT Actor Network Theory), envisagé comme « une série d'associations entre éléments hétérogènes ». Ce sera la nature du social à laquelle se rapportera l'exercice de sémiotique sociale proposé ici. La définition de l'espace en tant qu'objet de réflexion s'impose dans ce cas et c'est son caractère social que nous souhaitons rapporter dans les termes de l'auteur. Il sera en particulier intéressant de s'interroger sur le rôle des acteurs non humains, et plus précisément sur le rôle des objets ${ }^{5}$, dans l'agencement de l'espace. En soi, ce dernier sera traduit, et cela, en dernière analyse, sous la forme matérielle par le biais de laquelle nous pouvons trouver les vestiges d' « un mouvement qui se produit au cours du processus de groupement»(Latour). Nous nous proposons de suivre et relater le mouvement avec lequel on (re)fait l'espace, conçu comme un ensemble particulier de relations matérielles. Une fois empêché de se fixer, cet espace continue à être, en lui-même, un modulaire permanent d'hésitations, un espace-entre.

Déambulant par-entre «A Cidade dos Objectos» [La Ville des Objets] d'AAS : bref exercice de lecture $^{6}$

\section{Espaces vides et pleins}

Si l'on remonte à l'ancienne conception d'espace, selon laquelle celui-ci est une abstraction, produit de l'«activité configuratrice, opérative, de l'esprit rationnel et mathématique » (Jiménez, 2005: 46), José Jiménez indique que c'est «précisément parce qu'il manque de « figure », de forme que l'espace agit catégoriquement comme la borne conceptuelle qui permet de distinguer et de

\footnotetext{
${ }^{5}$ En tant qu'acteurs et dans la compréhension de Latour, ils sont définis à l'opposé d'une conception commune qui les conçoit en tant que simples supports de projections symboliques.

${ }^{6}$ Les images photographiques analysées concernent l'Exposition suivante : 1991. «A Cidade dos Objectos» [La Ville des Objets] Expo. Indi. pour le Centre Portugais de Design, Fondation Serralves Cat.

Nous avons pris en compte la série d'images de ladite Exposition, figurant dans la publication suivante : Silva, A.A. (2009).

Ensaio Sobre o Optimismo [catalogue d'exposition]. Porto: Serralves.
} 
délimiter toute forme ou figure (Idem : 47), en ajoutant, «mais également comme le contexte qui nous permet d'établir des distinctions entre le vide et le plein [soulignés par nous] ... » (Idem : 47).

Dans A Cidade dos Objectos [La Ville des Objets], d'Augusto Alves da Silva (AAS), les distinctions entre les vides et les pleins sont faites par l'action, sur les images des espaces (ou de ce que nous appelons espaces car ceux-ci permettent de délimiter des formes ou des figures) qui s'affirment réifiants de la concentration de figures de différentes natures matérielles (automobiles, bâtiments, immeubles urbains, arbres / végétation...). Une telle action gagne apparemment une signification sémiotique par opposition ${ }^{7}$ à l'action des espaces qui s'ouvrent à l'absence de cette même concentration. Nous pourrons attribuer aux premiers la désignation d'espaces pleins et aux derniers celles d'espaces vides.

Ce sont en particulier les espaces vides qui gagnent de l'importance sur les images d'AAS. Les routes sans trafic routier, les terrains vagues (contigus à une grande concentration d'immeubles urbains, dans l'un des cas), occupent littéralement une grande partie de l'espace des images. La production de A Cidade dos Objectos semble donc, en dernière analyse, être motivée par une interrogation sur le statut accordé généralement à l'espace, en tant que toile de fond ou scénario sur lequel se recoupent d'autres objets. Contrairement à la définition présentée par Jiménez (2005), les images d'AAS suggèrent, en vérité, l'assertion de l'espace par rapport aux autres signes. Dans la perspective de Charles Peirce, c'est la priméité du signe qui s'affirme, ainsi, l'indépendance des qualités de l'espace, ce qu'il est en lui-même. À en juger d'après ce que les images d'AAS suggèrent, l'espace est, en lui-même, tantôt un vide tantôt un plein qui devient l'objet visible du regard ${ }^{8}$. Ce n'est plus une catégorie abstraite, d'après une version géométrique et cosmologique, mais une représentation sensible qui, à son tour, permet de témoigner de l'urgence de l'espace lui-même.

Par analogie, nous pouvons également dire que dans Blow-Up, de Michelangelo Antonioni, nous assistons à un déplacement du sens reconnu, dans ce cas, au parc que Thomas (photographe professionnel dans le film), un tant soit peu par inadvertance, capte et recoupe avec sa caméra (voir Lefebvre, 2006 : 38-44). Investi au départ dans la reconstitution d'une espèce de narrative que la présence d'un couple dans le parc commence par stimuler - surtout lorsque l'analyse insistante des photographies, par Thomas, finit par s'ouvrir à la découverte subite de ce qui semble être des indices d'une mystérieuse forme (semi-occultée par la végétation entourant la clairière qui sert de scénario à la rencontre du couple photographié) non identifiable (S'agit-il d'une figure humaine ? Peut-on deviner un bras empoignant un révolver, probablement pointé sur le couple ? Ou est-ce plutôt un cadavre humain, en admettant également un enchaînement successif de différents stades d'une narrative supposée ?) - le photographe voit son intérêt pour la reconstitution et l'analyse de la narrative

\footnotetext{
${ }^{7}$ Rappelons-nous que chez Ferdinand Saussure, en particulier, la signification est bâtie grâce au caractère distinctif et oppositif des signes.

${ }^{8}$ La notion d'espace que les images d'AAS convoquent suppose une interrogation sur la vision géométrique de l'espace (vision abstraite de la nature et de ses formes). Jiménez (2005: 45) dit : «À première vue, l'espace est transparent, invisible, nous voyons les choses, les personnes, les objets, mais pas l'espace».
} 
s'estomper, à un certain moment, suite à sa fascination progressive pour les formes surprenantes qui se révèlent peu à peu à son regard. D'un fond scénique où une narrative supposée aurait eu lieu, le parc se transforme ainsi, comme cela figure sur les photographies, en une forme visible et autonome par rapport à son sens référentiel ou aux autres signes qui le prennent pour contexte de cette même narrative ${ }^{9}$. C'est comme si l'action à un certain moment était négligée par rapport aux temps morts désignés (temps morts) ${ }^{10}$, c'est-à-dire que c'est comme si, à un certain moment, nous assistions à la suspension du temps, à sa spatialisation absolue.

Les images d'AAS invitent à une lecture quelque peu similaire. Avant toute chose, la figure humaine est pratiquement éradiquée de l'espace de la représentation. Comme il s'agit au départ de photographies de paysages urbains (A Cidade dos Objectos), ce qui induirait peut-être l'expectative de voir représentées des «multitudes », c'est l'association commune de celles-ci à l'urbain, c'est dans la vérité des objets, du monde «naturel» et de l'espace lui-même pris pour objet en soi que les photographies s'occupent. Même dans les cas où les automobiles et les transports publics apparaissent représentés, en pose de circulation, ceux-ci sont mis en évidence en tant qu'involucres fermés sur euxmêmes et ne laissent rien apercevoir d'autre que des traits peu définis en leur intérieur. Dans l'un des cas en particulier, la captation d'un véhicule automobile à grande vitesse, bien que sur un plan relativement rapproché, se traduit par un effet de flou qui accentue l'impossibilité d'identifier clairement les formes, que ce soit de l'automobile elle-même ou de son intérieur.

La ville qu'AAS nous fait voir est donc une ville apparemment vide, proche des rues et des avenues qui, dans la partie initiale du film L'Homme à la Caméra de Dziga Vertov, sont présentées absolument nues, vides de gens, fantasmatiques. Cependant, tandis qu'avec Vertov, cela s'articule avec un jeu significatif déterminé, figuré par l'alternance entre le jour et la nuit, associé pour sa part, en dernière instance, à la connotation idéologique que le thème de l'endormissement et du réveil de la ville évoque à lui seul, avec AAS, l'endormissement et le réveil, la nuit et le jour, que le fait que la ville soit vide peut éventuellement suggérer, semblent se fondre en une seule atmosphère étrange. Toutes les images sont captées en pleine lumière du jour, ce qui au départ, pourrait faire s'attendre à les voir remplies, pleines de figures et d'objets donnant une impression de mouvement et d'agitation. Au lieu de cela, la ville s'exhibe semi-endormie, presque inanimée, à l'exception ici et là de véhicules (sur deux des sept images de la série analysée) captés au passage. Une ville fantasmatique ? S'ajoute à ce sentiment d'étrangeté car des images (publicités outdoors) s'inscrivent à leur tour dans les images, en véhiculant ainsi la représentation de figures humaines, en même temps que celles-ci se trouvent pratiquement absentes de l'espace territorial représenté.

\section{Liaisons, coupes et interférences imprévisibles}

\footnotetext{
${ }^{9}$ Lefebvre (2006), à propos de sa propre analyse du film, suggère le passage du lieu au paysage, c'est-à-dire du « setting » au «landscape ».

${ }^{10}$ Lefebvre (2006) associe, précisément, lesdits temps morts à la notion de passage.
} 
Dans A Cidade dos Objectos, l'espace de représentation est reparti sur chaque image, grosso modo, en deux parts plus ou moins équitables, une inférieure, donnant lieu à la représentation du «sol » et l'autre supérieure, donnant lieu à la représentation du ciel. À mi-chemin, entre les limites inférieure et supérieure de l'image, s'inscrit une «bande », distendue dans le sens horizontal et latéral de l'image, plus ou moins épaissie par différentes formes : arbres, véhicules, outdoors, pièces de mobilier urbain, bâtiments et, en particulier, immeubles, poteaux et panneau de circulation, etc. Il s'agit d'une « bande » dont le sens d'horizontalité est ponctuellement contrarié par les lignes de force configurées par certains bras de route représentés, d'une part, ou par l'identification d'un certain point de fuite (dans le cas de la perspective linéaire sous-jacente à la représentation sur deux des images) de l'autre. Ces lignes de force agissent comme des coupes dans la prévisibilité que la direction unique apparente (l'horizontalité) présupposerait, en introduisant de petites déviations, voire des hésitations dans la conduite du regard. D'une manière ou de l'autre, les images invitent à l'identification de seuils qui en même temps qu'ils se séparent (le sol ou la terre du ciel) se transforment en formes de contiguïtés et d'une certaine indéfinition.

Avant tout, nous pouvons dire que s'il y a une ligne d'horizon identifiable, elle correspondra, précisément, au seuil où la terre et le ciel se touchent. Comme il ne s'agit pas d'une ligne d'horizon désobstruée et par conséquent capable de suggérer véritablement le prolongement du regard sur la distance et les espaces infinis, l'effet d'approximation au regard, l'avancée de l'horizon sur ce côté de l'image s'impose avec une plus grande force. Se convertissant ainsi en une expérience tactile, le regard introduit, à son tour, l'observateur à une contiguïté matérielle déterminée avec l'image ellemême, en stimulant $l$ '«expérience» de la matérialité à laquelle ses formes se réfèrent. Le sol représenté est, en particulier, mis en évidence dans sa diversité formelle et matérielle. Tantôt chaussée goudronnée, tantôt chaussée en pierres, tantôt trottoir dallé ou terreux, tantôt sol fleuri ou en terre, tantôt bas-côté en fleurs ou simplement avec de la végétation, plus ou moins « spontanée »... C'est sur une telle diversité que s'opèrent des marques de délimitation : murs, bas-côtés, grillages, palissades...

La Nature évoquée se dédouble pour sa part en une double fonction. D’une part, sous forme de jardin, elle donne une visibilité à certaines marques distinctives et de séparation (ronds-points, marques de séparation entre voies de la route, bas-côtés...). De l'autre, elle se montre capricieuse, imprévisible, voir excessive dans son action. Parce qu'elle se révèle éclosant parmi les fissures du sol tissé avec des pierres, parce qu'elle s'interpose à la visibilité d'une autre manière dont l'aspect (dessiné pour le regard) se voit ainsi semi-occulté (cas d'une image dans laquelle l'outdoor représenté est partiellement caché par un arbre) mais aussi parce qu'elle s'étend simplement dans tout l'espace «libre » d'édification, avivant ainsi l'idée de Nature avec laquelle la ville a toujours cohabité, et cela d'ailleurs de forme tensive (sur l'une des images, il est possible de voir un grand terrain entièrement fleuri, au premier plan, contigu à une concentration d'immeubles urbains au fond). 
Pourrons-nous parler ici d'« énonciations pédestres » à la manière de Michel Certeau (2000 [1990]), en envisageant les pratiques «déviantes » que les formes et les objets dans les images personnifient? L'auteur considère qu'aux formes et sens prévisibles qui orientent l'action dans le cadre de l'espace urbain s'opposent des modes particuliers des individus de s'approprier ce même espace, à l'image de ce qui arrive avec les usages de la langue par rapport à l'ensemble de règles qui définissent la langue elle-même. Chacun est libre, jusqu'à un certain point, de s'arrêter ou de marcher, de suivre une direction ou une autre, voire, en dernière analyse, de « marcher ou pas sur le gazon »...

Les images d'AAS semblent rendre compte d'une espèce de signification des «pas perdus » et silencieux, en tenant compte non pas des pas à proprement humains, mais de la simple action matérielle et objectuelle comme ce qui fait de l'espace ce qu'il est en lui-même: un perpétuel « devenir-autre » (Deleuze et Guattari), un enchaînement infini d' «associations » (Latour)... L'action suggérée est silencieuse et survient dans un jeu entre le visible et l'occultation.

\section{Du visible et de l'occultation}

Le contrepoint entre le ciel et la terre que les images semblent mettre en évidence pourrait, au départ, suggérer une opposition sémantique : le vide du ciel d'une part et le plein de la «ville» de l'autre. Cependant, si le ciel est immédiatement associé à l'infinitude de l'espace, ainsi qu'au caractère éthéré de sa nature matérielle, les deux dimensions étant pour leur part suggestives de l'idée de vide (voir Sauvageot), il n'est pas aussi évident que dans le cas des images d'AAS, il soit possible d'associer à la terre, avec la même facilité, ce qui serait le contraire de cela : espace fini et délimité, densité, profusion et concentration de formes ou de figures matérielles.

En vérité, sur lesdites images, avec le plein de la ville, traduit sous les formes visibles les plus diverses (bâtiments, arbres, véhicules, publicités...), habite l'absence, le vide des personnes, qui, comme cela a déjà été indiqué, ne se laissent deviner que sous la forme de traits semi-occultés à l'intérieur des véhicules ou de simulacres, figurés sur les outdoors qui s'exhibent en face des voies de circulation. En dernière analyse, il semblerait que ce soit la propre condition de l'existence contemporaine dans les villes qui est apparemment accentuée. Dessinées pour la circulation rapide, en automobile ou dans un autre type de transport, les villes que les images évoquent empêchent le contact direct du corps avec le territoire, ainsi que sa fixation prolongée. Les images en analyse semblent également suggérer la quasi-impossibilité de captation du passage en soi, par voie photographique. En d'autres termes, ce sont les «pratiques pédestres d'énonciation», auxquelles Michel de Certeau se réfère, qui se présentent ainsi menacées.

$\mathrm{Au}$ lieu d'une narration sur les «pas perdus », il est important pour AAS de donner de la visibilité aux passages inconnus, à ce dont on devine qu'il est passé (ou passera) sans laisser de traces, c'est-à-dire à ce qui semble perpétuellement condamné à l'oubli, au bruit qui, à tout moment, peut se transformer en silence. Les objets sont ce qui demeure apparemment, mais également le monde «naturel », considérés comme des formes matérielles non humaines. Ce sont les seigneurs de la ville 
et de l'espace visible. En nous rendant la conscience du regard, ils constituent l'altérité de notre être, en même temps qu'ils servent de preuve à notre propre ontologie (voir Merleau-Ponty, 2001 [1945]).

En dernière instance, les objets et les différentes formes représentés sur les images d'AAS inscrivent la présence humaine sous la figure de l'observateur qui, situé en dehors de l'image, est présupposé dans la distance du regard à la cible. Merleau-Ponty (2001 [1945]), à propos de l'unité du processus perceptif, mentionne la "distance optimale" en deçà et au-delà de laquelle la perception devient confuse :

\footnotetext{
«Pour chaque objet comme pour chaque tableau dans une galerie de peinture, il y a une distance optimale d'ù il demande à être vu, une orientation sous laquelle il donne davantage de lui-même: en deçà et au-delà nous n'avons qu'une perception confuse par excès ou par défaut, nous tendons alors vers le maximum de visibilité et nous cherchons comme au microscope une meilleure mise au point, elle est obtenue par un certain équilibre de l'horizon intérieur et de l'horizon extérieur... » (Merleau-Ponty, 2001 [1945]: 348).
}

Dans A Cidade dos Objectos en particulier, nous assistons à une sensation simultanée de proximité et d'éloignement qui semble menacer, dans certains cas, ladite « distance optimale », c'està-dire, comme l'indique Merleau-Ponty (2001 [1945]), l'équilibre entre «l'horizon intérieur et l'horizon extérieur ». D'une part, la ligne de l'horizon apparaît reculée à partir du premier plan de l'image, s'ouvrant à l'infiniment lointain, en même temps qu'elle justifie un exercice télescopique du regard. De l'autre, le « sol » tel qu'il est représenté, en personnifiant le premier plan, semble avancer sur la position de l'observateur, au-delà de l'espace de la représentation, invitant cette fois à la vision microscopique. L'observateur se voit ainsi renvoyé à un va-et-vient permanent d'hésitations.

\section{Note finale}

La ville que les images de A Cidade dos Objectos décrivent au départ se présente dans sa nature hybride, indéfinissable, décaractérisée. La cohabitation (que ce soit dans chaque image ou dans la relation entre les images) entre les bâtiments de type différencié (urbains et non urbains), entre les routes, d'une part, et les «îles » de végétation, plus ou moins spontanée, de l'autre, entre les espaces d'une certaine façon comprimés (dont les propres immeubles, compris comme produit de la compression de l'espace habitable, sont des exemples) et les espaces plus amples voire vagues, permet de s'interroger sur la propre notion de ville à laquelle nous nous référons. En vérité, ce qui est mis en évidence, ce sont les espaces limitrophes, la rencontre des objets (compris en tant que formes non humaines et non naturelles) avec la Nature, de l'urbain avec les derniers reliquats de la ruralité. La Nature que les images évoquent ne cesse cependant de se configurer comme un espace à coloniser, occupé par les objets qui se répandent sans fin, en avançant aussi bien en deçà qu'au-delà de l'horizon possible. Les objets dont l'action menace de s'étendre se présentent, quant à eux, destitués de toute fonction symbolique, c'est-à-dire qu'ils s'imposent dans leur fonctionnalité absolue (comme c'est le cas des immeubles, des véhicules...). Ironiquement, ce sont les images représentées sur les images (publicité outdoors), ainsi que la signalétique et autres (stations-service avec identification du logotype 
de la marque Galp) qui semblent constituer les seules marques de référenciation culturelle et symbolique. Cela veut dire que l'espace territorial représenté se révèle comme un « non-lieu » (Marc Augé), un lieu de passage contraire à la fixation de l'histoire, de la relation ou de l'identité. La Ville qu'AAS nous invite à voir est, en dernière analyse, pratiquement inidentifiable, son aspect pouvant être confondu avec celui de n'importe quelle ville.

Sur les images, l'espace vide qui entoure ou recoupe les objets, qu'il s'agisse d'un espace qui a fait l'objet d'une intervention (les routes, les tapis en fleurs ...) ou d'un espace «naturel » (les terrains vagues), produit un sentiment accentué d'isolement et d'incommunicabilité. Comme il ne s'agit pas à proprement parler d'un espace-libre, une fois sa condition tensive mise en évidence, l'espace dont on tient compte assume un caractère oppressif et quelque peu pathologique. Largement exhibé, le ciel, en raison de son caractère véritablement désobstrué accentue, à son tour, la sensation trompeuse et paradoxale de distension-contraction de l'espace sur terre.

Pour finir, il nous reste à conclure que l'analyse de A Cidade dos Objectos, de Augusto Alves da Silva, nous semble expliquer la manière dont des entités matérielles de nature hétérogène se constituent elles-mêmes comme des entités sémiotiques. Les images mettent en évidence la «transgression des frontières » à la manière d'Haraway ou l'infinité d' " associations », à la manière de Latour. Dans un cas comme dans l'autre, c'est l'ontologie de l'espace, indice de la condition de notre existence contemporaine, qui est ainsi pris pour objet d'interrogation...

\section{Références bibliographiques :}

Augé, Marc (1993). Los «No Lugares». Espacios del Anonimato - Una Antropologia de la Sobremodernidad. Barcelonne : Gedisa Editorial.

Certeau, Michel (2000) [1990]. A Invenção do Cotidiano - Artes de Fazer. Petrópolis : Editora Vozes.

Chomsky, N. (1965). Aspects of the Theory of Syntax. Cambridge: MA MIT Press.

Deleuze, Gilles e Guattari, Felix (s/d). O Anti-Édipo. Capitalismo e Esquizofrenia. Lisbonne : Assírio \& Alvim.

Gaines, E. (2006). Communication and the Semiotics of Space. Journal of Creative Communications 1:2. London : Sage Publications, 173-181.

Hall, E. (1966). The Hidden Dimension. New York : Routledge. 
Haraway, D. (1987). A manifesto for cyborgs : science, technology, and socialist feminism in the 1980's. Australian Feminist Studies, 4, 1-41.

Jiménez, José (2005). Pensar o espaço. In Bragança de Miranda, José e Prado Coelho, Eduardo, Espaços (Revista de Comunicação e Linguagens, n 34 e 35). Lisbonne : Relógio D'Água Editores.

Latour, B. (1990). Drawing things together. In M. Lynch \& S. Woolgar (eds.) Representation in Scientific Practice. Cambridge : MA MIT Press, 19-68.

Latour, B. (2006). Como prosseguir a tarefa de delinear associações?. Configurações, $n^{\circ}$ 2, 11-27.

Lefebvre, H. (1991) The Production of Space. Cambridge : MA Trans Blackwell.

Lefebvre, Martin (Ed.) (2006). Landscape and Film. Londres : Routledge.

Lévy, F. P. \& Segaud, M. (1983). Anthropologie de l'espace. Paris. Centre Georges Pompidou.

Merleau-Ponty (2000) [1964]. O Visível e o Invisível. São Paulo: Editora Perspectiva.

Roderick, I. (2001). Bodies, Materials, Practice: Habiting Space and the Representational Limits of Latour's Semiotics of Assemblies. Social Semiotics, Vol. 11, $N^{o} 3,343-360$.

Sauvageot, Anne (1987). Figures de la Publicité, Figures du Monde. Paris: PUF.

Silva, A.A. (2009). Ensaio Sobre o Optimismo [catalogue d'exposition]. Porto : Serralves.

Thornberg, J. M. (1979). Topogénesis Dos. Ensayo sobre la Naturaleza Social del Lugar. Barcelone : Ediciones Oikos. 